\title{
Intermédialités
}

Histoire et théorie des arts, des lettres et des techniques

Intermediality

History and Theory of the Arts, Literature and Technologies

\section{Traduction, re-traduction, hétérolinguisme dans la littérature postcoloniale italienne}

\section{Lucia Quaquarelli}

Numéro 27, printemps 2016

traduire

translating

URI : https://id.erudit.org/iderudit/1039812ar

DOI : https://doi.org/10.7202/1039812ar

Aller au sommaire du numéro

Éditeur(s)

Revue intermédialités (Presses de l’Université de Montréal)

ISSN

1920-3136 (numérique)

Découvrir la revue

Citer cet article

Quaquarelli, L. (2016). Traduction, re-traduction, hétérolinguisme dans la littérature postcoloniale italienne. Intermédialités / Intermediality, (27).

https://doi.org/10.7202/1039812ar
Résumé de l'article

La littérature postcoloniale italienne interroge le statut du texte tant sur le plan de son appartenance à un auteur et à une tradition ou canon littéraires nationaux, que sur le plan de l'homogénéité de la langue, des rapports de pouvoir entre les langues (et les littératures) et les stratégies de traduction. Le cas de Immigrato (1990) de Mario Fortunato et Salah Methnani, ainsi que les romans de Ubah Cristina Ali Farah, Madre piccola (2007) et Il comandante del fiume (2014), nous permettront de discuter ces hypothèses. 


\title{
Traduction, re-traduction, hétérolinguisme dans la littérature postcoloniale italienne
}

\author{
LUCIA QUAQUARELli
}

«Il traduttore è la vittima di difficoltà insite alla natura stessa del linguaggio; il lavoro del traduttore non fa che renderle manifeste. »

Benvenuto Terracini, $1983^{\mathrm{I}}$

ne précision avant tout : j'entends par « littérature postcoloniale italienne », ou « littérature italienne de la migration », ce corpus, hétérogène et à frontières variables, qui a pris forme en Italie dans les trente dernières années ${ }^{2}$ comme effet d'un flux inédit de migrants vers le pays. Il s'agit donc de textes (romans, nouvelles, poèmes) d'auteurs non-italiens, écrits en langue italienne, et pour un public et un circuit éditorial italiens. Les deux appellations, «postcoloniale» et « migrante », très

${ }^{\mathrm{I}}$ \& The translator is the victim of the difficulties inherent to the very nature of language; the work of translation only makes the latter manifest » (notre traduction).

${ }^{2}$ Pour approfondir le sujet, consulter : Daniele Comberiati, Scrivere nella lingua dell'altro. La letteratura degli immigrati in Italia (1989-2007), Bruxelles, Peter Lang, 2010; Silvia Contarini, Giuliana Pia et Lucia Quaquarelli (dir.), « Coloniale e Postcoloniale nella letteratura italiana degli anni $2000 »$, Narrativa, ${ }^{\circ}$ 33/34, 2012; Roberto Derobertis (dir.), Fuori centro. Percorsi postcoloniali nella letteratura italiana, Roma, Aracne, 20Io; Armando Gnisci(dir.), Nuovo planetario italiano. Geografia e antologia della letteratura della migrazione in Italia e in Europa, Troina, Citta aperta, 2006; Cristina Lombardi Diop et Caterina Romeo, L'Italia postcoloniale, Milano, Mondadori, 20I4; Maria Cristina Mauceri et Maria Grazia Negro, Nuovo immaginario italiano. Italiani e stranieri a confronto nella letteratura italiana contemporanea, Roma, Sinnos, 2009; Chiara Mengozzi, Narrazione contese. Vent'anni di scritture italiane della migrazione, Roma, Carroci, 2013; Fulvio Pezzarossa et Ilaria Rossini (dir.), Leggere il testo e il mondo, Bologna, Clueb, 2oII; Lucia Quaquarelli(dir.), Certi confini. Sulla letteratura italiana dellimmigrazione, Milano, Morellini, 2oıo; Lucia Quaquarelli, Narrazione e migrazione, Milano, Morellini, 2or5; Franca Sinopoli (dir.), Postcoloniale italiano. Tra letteratura e storia, Aprilia, Novalogos, 20I3. 
problématiques et très courantes à la fois, ont fini par se superposer et par se confondre dans les réflexions des chercheurs tout comme chez les critiques littéraires, les libraires et les lecteurs 3 , dessinant au fil des années une « zone » littéraire à part, distincte de la production nationale «non migrante » et pourvue d'un certain nombre de traits communs suffisamment caractérisants pour produire une « communauté 4 » et tracer le profil d'un phénomène culturel largement reconnu et modestement étudié : partage de thèmes et de formes littéraires; forte hypothèque testimoniale; même situation d'énonciation; mêmes normes d'accès au marché éditorial...

L'utilisation presque synonymique de deux définitions - postcoloniale et migrante - pourvues d'une spécificité épistémologique, politique et culturelle qui semble les distinguer une fois pour toutes, outre le fait qu'elle constitue un fait culturel - qui doit en tant que tel être pris en compte et interrogé —, me paraît fondée si l'on considère un certain nombre d'éléments.

Premièrement, les flux migratoires à la suite desquels ces auteurs « migrants » sont arrivés en Italie s'insèrent dans un cadre historique et géopolitique de continuité, de prolongement et d'élargissement des stratégies et des dispositifs coloniaux : les migrants sont les nouveaux sujets coloniaux s et les politiques migratoires européennes, politiques d'inclusion sélective et différentielle, re-proposent, à l'échelle interne, les mêmes dynamiques d'exploitation et de ségrégation qu'à l'ère impérialiste, continuant à réguler la cohabitation des peuples de manière profondément et violemment hiérarchique.

${ }^{3}$ Le débat sur ces « étiquettes » et « définitions » a été très animé en Italie, privilégiant tout d'abord l'occurrence « littérature italophone » et défendant par la suite, successivement et de manière parfois problématique, les variantes « littérature de l'immigration », « littérature migrante », «littérature de la migration » ou «littérature postcoloniale ». Pour la reconstitution de ce débat, je renvoie à Giuliana Benvenuti, «Letteratura della migrazione, letteratura postcoloniale, letteratura italiana. Problemi di definizione », dans Pezzarossa et Rossini, 20II, p. 247-260, ainsi qu'au chapitre «Definizioni e problemi » dans Quaquarelli, 2015, p. 9-38.

4 J'utilise ici le terme « communauté » dans un sens proche de celui de la sociolinguistique : un ensemble de locuteurs qui ont en commun un même comportement à l'égard d'une langue et de ses normes d'usage. À ce sujet, voir, entre autres, Jack K. Chambers, Sociolinguistic Theory [1995], Oxford, Wiley-Blackwell, 2008; Lesley Milroy et Matthew Gordon, Sociolinguistics. Method and Intepretation, Oxford, Blakwell, 2003; Giorgio Raimondo Cardona, Introduzione alla sociolinguistica, Novara, De Agostini, 2009.

5 Voir à ce propos Étienne Balibar, Nous, citoyens d'Europe? Les frontières, l'État, le peuple, Paris, La Découverte, 20or; Sandro Mezzadra, La condizione postcoloniale. Storia e politica nel presente globale, Verona, ombre corte edizioni, 2008. 
Deuxièmement, les auteurs qui nous occupent ici ont avec la langue italienne, tous et indépendamment de leur pays d'origine, un rapport très similaire à celui vécu par les écrivains colonisés/coloniaux, car l'italien est pour eux une langue imposée. Et même, il est une langue doublement imposée : d'abord par les rapports de pouvoir mondiaux qui ont poussé les auteurs à la fuite et les ont contraints à l'exil; puis par la « république mondiale des lettres », cette organisation eurocentrique qui, comme nous le rappelle Pascale Casanova ${ }^{6}$, fait de l'italien une langue véhiculaire, à la différence du wolof et du somali, par exemple, permettant aux textes leur insertion (potentielle) dans un circuit littéraire mondial.

De plus, les textes eux-mêmes témoignent d'un air de famille (en grande partie prescrit par les dispositifs de l'industrie culturelle italienne) quant aux thèmes, modes et formes littéraires qui rendent problématique toute distinction « géo-littéraire » et « socio-historique ». Il est ainsi très difficile, il me semble, de ne pas lire l'intégralité du corpus dans une perspective postcoloniale, adoptant une posture critique qui, puisqu'elle traverse frontières et pays, nous aide à mesurer combien les pratiques de subjectivation/assujettissement en œuvre dans ces textes sont profondément liées aux structures de pouvoir dérivées tant des politiques coloniales que de l'actuelle organisation globale du monde qui en est l'héritière. C'est pourquoi je trouve tout particulièrement intéressant la définition de « postcolonialité indirecte » proposée par Cristina Lombardi Diop et Caterina Romeo, qui nous permet d'élargir les mailles de la notion et de reconnaître que le fait que de nombreux auteurs immigrés ne proviennent pas d'anciennes colonies italiennes ne modifie pas, en substance, la nature et les modalités de leurs rapports avec les Italiens, la langue italienne, le canon littéraire et l'industrie culturelle nationale et internationale, puisque, écrivent Diop et Romeo, « en Italie la dimension post-nationale est une composante essentielle de la condition postcoloniale $\gg$.

C'est à partir d'un tel ensemble de mouvements et de transitions épistémologiques, de glissements et d'enchaînements entre histoire coloniale et discours colonial - conçu comme discours de la contemporanéité qui impose une révision des catégories et des méthodes d'analyse critique - , que je vais essayer d'interroger la place (et le rôle) de la traduction dans les rapports de force entre les langues (les hommes, les cultures) en œuvre (et en scène) dans certains de ces textes.

\footnotetext{
${ }^{6}$ Voir Pascale Casanova, La république mondiale des lettres [1999], Paris, Seuil, 2008.

7 Cristina Lombardi Diop et Caterina Romeo, 20I4, p. I4.
} 
Commençons par une citation quelque peu provocatrice, mais qui permet d'introduire le débat autour de la langue : «J'ai perdu trop de temps - écrit Dany Laferrière - à commenter le fait que j'écris en français. Et à débattre du fait que ce ne soit pas ma langue maternelle $e^{8} \gg$.

Les écrivains postcoloniaux qui «adoptent» la langue du colon dans la création littéraire non seulement parlent, au moins, une autre langue et écrivent à partir d'une position de bi(ou pluri)linguisme, mais ils écrivent aussi souvent, comme le souligne Samia Mehrez, à partir d'un «bilinguisme radical 9 », qui évoque non seulement deux (ou plusieurs) cultures linguistiques différentes (étrangères), mais également deux (ou plusieurs) cultures en conflit. Ce bi(ou pluri)linguisme comporte en soi l'expérience traumatique du refoulement et de l'abandon forcés de la languemère - à laquelle on s'est référé en recourant aux termes d' « arrachement » (Fanon), de « déchirure » (Glissant), de «dissociation » (Derrida) - , ainsi que celle, également traumatique et violente, de l'imposition (directe ou indirecte, patente ou latente) d'une langue étrangère.

Or, une grande partie de la réflexion théorique - à partir du texte fondateur de Ashcroft, Griffiths et Tiffin, The Empire Writes Back, en passant par la relecture d'Haroldo de Campos du Manifesto Antropofago de Oswald de Andrade et par Location of Culture de Homi Bhabha, jusqu'aux publications les plus récentes ${ }^{\mathrm{IO}}$ concorde avec l'idée que le travail mené par les écrivains postcoloniaux sur la langue littéraire est un des dispositifs qui fondent la portée oppositive et contre-narrative de leurs écritures, le lieu du talking back, l'espace à partir duquel non seulement les écrivains obtiennent un certifié d'existence (personnelle et littéraire), mais grâce auquel ils prennent la parole pour renverser les lignes du pouvoir. Écrire dans la langue du colon permet ainsi de lui ôter son pouvoir, le pouvoir sur la langue (la maîtrise de la langue) et le pouvoir sur les hommes, ce qui revient à écrire au nom de la liberté.

\footnotetext{
${ }^{8}$ Dany Laferrière, « Je voyage en français », dans Michel Le Bris, Jean Rouaud (dir.), Pour une littérature-monde, Paris, Gallimard, 2007, p. 87.

9 Samia Mehrez, «Translation and Postcolonial Experience », dans Lawrence Venuti (dir.) Retbinking Translation, London/New York, Routledge, 1992, p. 120.

ro Bill Ashcroft, Gareth Griffiths et Helen Tiffin, The Empire Writes Back [1989], London/New York, Routledge, 2002; Haroldo de Campos, « Da razão antropofágica: diálogo e diferença na cultura brasileira », Boletim bibliográfico, São Paulo, $\mathrm{n}^{\circ}$ 44, janvier-décembre 1983 , p. I07-I25; Homi Bhabha, Location of Culture, London/New York, Routledge, 1994.
} 
L'essentielle fonction de la langue comme instrument de pouvoir réside, écrivent Ashcroft, Griffiths et Tiffin ${ }^{\text {II }}$, dans la possibilité qu'ont les écrivains d'abroger le privilège de la « maîtrise » linguistique du peuple colonisateur et, tout à la fois, dans le processus de réappropriation linguistique qu'ils accomplissent par le biais de la déconstruction et de la transformation de cette même langue. Car la langue du colon est souvent remaniée, réinventée, absorbée, dévorée, et la «langue-mère » peut à nouveau prendre la parole, à l'intérieur de la langue de l'autre, révoquant de cette façon son authenticité, son essentialité et sa centralité.

Cette réappropriation de la langue inaugure, d'un côté, un processus d' « autonomisation de la langue ${ }^{\mathrm{I2}}$ » qui garantit à celle-ci un statut trans-national (pré- ou post-national) et met en cause toute frontière géopolitique rigide. La langue se trouvant alimentée d'aventures culturelles, sociales et littéraires différentes, elle peut se faire hybride, créole, dans un sens proche de celui indiqué par Édouard Glissant ${ }^{13}$.

D’autre part, cette réappropriation semble fonder la possibilité de la subversion des relations de pouvoir entre les langues (et les hommes) par la force d'un subtil et constant «sabotage » interne de l'ordre et de l'homogénéité supposés de la langue dominante, mené grâce à un certain nombre de pratiques de déplacement, déviance, citation, emprunt, calque, invention... qui permettent l'entrée, et l'affirmation, de la langue des subalternes au sein du périmètre de la langue dominante. Il s'agirait en somme d'une forme d'agôn linguistique, de « guérilla » linguistique (très loin cette fois de l'idée qu'a Glissant de l’hybridation comme relation) menée au nom de la liberté.

Ainsi se prononce Salman Rushdie dans Imaginary Homelands:

I hope all of us share the view that we can't simply use the language in the way the British did; that it needs remaking for our own purposes. Those of us who do use English do so in spite of our ambiguity toward it, or perhaps because of that, perhaps because we can find in the linguistic struggle a reflection of other struggles taking place in the real world, struggles between the cultures [...]. To conquer English may be to complete the process of making ourselves free ${ }^{\mathrm{I} 4}$.

Écrire dans la langue de l'autre ne signifie donc pas abdiquer sa propre langue, dit Rushdie, mais conquérir l'autre langue, conquérir l'Autre.

II Ashcroft, Griffiths et Tiffin, 2002, p. 37.

${ }^{12}$ Gilles Deleuze et Félix Guattari, Mille plateaux, Paris, Éditions de Minuit, 1980.

${ }^{13}$ Édouard Glissant, Introduction à une poétique du divers, Paris, Gallimard, 1996, p. 4I.

I4 Salman Rushdie, «Imaginary Homelands », dans Rushdie, Imaginary Homelands. Essays and Criticism 1981-199I, London/New York, Granta Books/Penguin Books, 1992, p. 17. 
L'écriture française nous "livre" à l'autre, mais on se défendra par l'arabesque, la subversion, le dédale, le labyrinthe, le décentrage incessant de la phrase et du langage, de manière que l'autre se perde comme dans les ruelles de la casbah ${ }^{15}$.

Or, il vaut mieux dire tout de suite que, sur cette question, les auteurs postcoloniaux écrivant en langue italienne, pour leur part, s'expriment beaucoup plus en faveur de la reconnaissance de la valeur interculturelle et interlinguistique de la langue littéraire plutôt qu'en termes de conquête, ré-écriture et revanche. Dans leurs déclarations, l'italien semble rester une langue moins menaçante que le français et l'anglais, marqué par une hospitalité presque protocolaire. Ce serait en ce sens une langue que l'on aborde par voie « naturelle ${ }^{16} »$, ou encore une langue « adoptive ${ }^{17} »$.

Cependant, il s'agit d'une position problématique, que l'on doit lire à partir de la rigide grille de sens (et à fonction sociale) qui caractérise depuis le début l'expérience italienne. Une grille dessinée par l'industrie culturelle (éditeurs, associations, écoles) pour répondre à des exigences avant tout d'ordre éducatif et culturel (et non littéraire) : les écrivains italiens de la migration sont depuis le début, aussi, des médiateurs culturels, et leurs textes, des laboratoires d'interculturalité, ce qui limite, de manière programmatique, la portée transgressive et conflictuelle de ces écritures.

Malgré les positions divergentes des auteurs, il importe de relever que, dans cette supposée bataille - ou rencontre - entre les langues et à l'intérieur des langues, se glisse la question de la traduction, question qui traverse la réflexion postcoloniale à plusieurs niveaux. Le premier - celui indiqué par Homi Bhabha - est celui de la traduction comme «tiers lieu », comme espace «entre-deux »: la traduction se présente comme figure de la différence culturelle et, à la fois, comme possibilité de

\footnotetext{
is Abdelwahab Meddeb, cité dans Jean Dejeux, Situation de la littérature maghrébine de langue française, Alger, OPU, 1982, p. I03-IO4.

${ }^{16}$ Je pense à Igiaba Scego quand elle déclare : « La langue dans laquelle j'écris est l'italien : il ne s'agit pas d'un choix, mais du cours naturel des choses » (notre traduction de : « la lingua in cui scrivo è l'italiano, non per scelta, ma per corso naturale »). « Relazione di Igiaba Scego », Eks E tra, associazione interculturale, premio letterario, database racconti e poesie, atività, 2004, www.eksetra.net/studi-interculturali/relazione-intercultura-edizione-2004/relazione-di-igiabascego/ (consultation le I7 janvier 20I7).

${ }^{17}$ L'italien comme langue d'adoption (et d'élection), dont parle Serge Vanvolsem. Serge Vanvolsem, «Dagli elefanti a nonno Dio. Il rinnovo del codice linguistico italiano con le scritture migranti », dans Pezzarossa et Rossini, 2oII, p. 3.
} 
négociation culturelle. En ce sens, la traduction participerait de la "nature performative" de la communication culturelle, elle serait une langue in actu et non in situ $^{18}$.

Toujours dans cette perspective, les écritures postcoloniales seraient conçues comme des écritures de la traduction, des écritures-traductions, dont la marque essentielle serait de défier les géographies linguistiques historiquement élaborées sur une base nationale; redonner voix à la langue-mère de l'auteur (et d'une communauté) là où elle avait été réduite au silence; ré-écrire les relations de pouvoir entre les langues (et les hommes); et (encore et surtout) pratiquer, performer (donc imaginer) la transculturalité.

La traduction serait donc un mode de génération textuelle capable de mettre à l'œuvre la porosité entre les langues et les cultures. Les écrivains postcoloniaux seraient quant à eux, comme l'a plusieurs fois répété Salman Rushdie, des «translated men », des hommes traduits, des hommes condamnés à vivre « across the world ${ }^{19}$ » et capables précisément pour cela de créer des « patries imaginaires » (imaginary homelands), c'est-à-dire un cadre esthétique et conceptuel «différé », et en grand partie métaphorique (dit toujours Rushdie), au sein duquel relire, ré-écrire et re-configurer la réalitéeo.

La traduction serait alors aussi la grande métaphore de la position (historique, politique et épistémologique) postcoloniale, à savoir la figure de cet espace/temps de relecture, de « traduction », qui s'ouvre après et au-delà de l'entreprise impérialiste et qui se fonde sur une critique définitive du colonialisme, traversant les disciplines et s'attachant à retrouver la voix de l'autre, à re-donner la voix (sa voix) à l'autre.

Mais la traduction serait également, si on avance encore dans la réflexion, un fait et un effet interne propre non seulement à une langue (littéraire), mais à toute langue : le signe, la marque profonde d'une hétérogénéité constitutive de toute langue (et de toute culture). Le critique serait alors appelé à reconnaître et à déconstruire la charpente fictive et historiquement située de toute unité linguistique, et à dévoiler les rapports de force qui l’ont élaborée et qui continuent à la renouveler.

À cet égard, je voudrais, rapidement, parcourir deux cas de traduction « interne » au sein de la production postcoloniale italienne.

${ }^{18}$ Homi K. Bhabha, The Location of Culture, London, Routledge, 1994, p. 355.

I9 Rushdie, 1992, p. 17.

${ }^{20}$ Salman Rushdie revient fréquemment sur ces idées. Voir en particulier Rushdie, 1992; Salman Rushdie, Shame, London, Cape, 1985; Salman Rushdie, «Gunter Grass », dans Rushdie, 1992, p. 273-28I; Salman Rushdie, Satanic Verses, London, Cape, 1988. 
Le premier cas est celui des écritures «à quatre mains », qui ont marqué surtout les premières années de la production migrante, à savoir des textes qui présentent, côte à côte, un auteur « migrant » et un auteur « sédentaire ». On a ici affaire à une typologie d'écriture, disons-le tout de suite, qui se positionne beaucoup moins du côté de la co-auctorialité et de l'écriture collective que de celui de la transcription, de la traduction ou encore de l'appropriation.

Il s'agit en fait de textes fondés, pour la plupart, sur une ambiguïté de fond : celle d'une auctorialité patente qui est accordée à l'immigré, mais d'une autorité linguistique et littéraire garantie et revendiquée par l'autochtone.

Dans les ouvrages des années 1990, en fait, le nom qui apparaît à titre d'auteur est celui de l'immigré, tandis que le deuxième, l'autochtone, s'il est visible en couverture, est précédé de « a cura $d i^{2 \mathrm{I}} »$, ou « dirigé par » (voir la figure I).
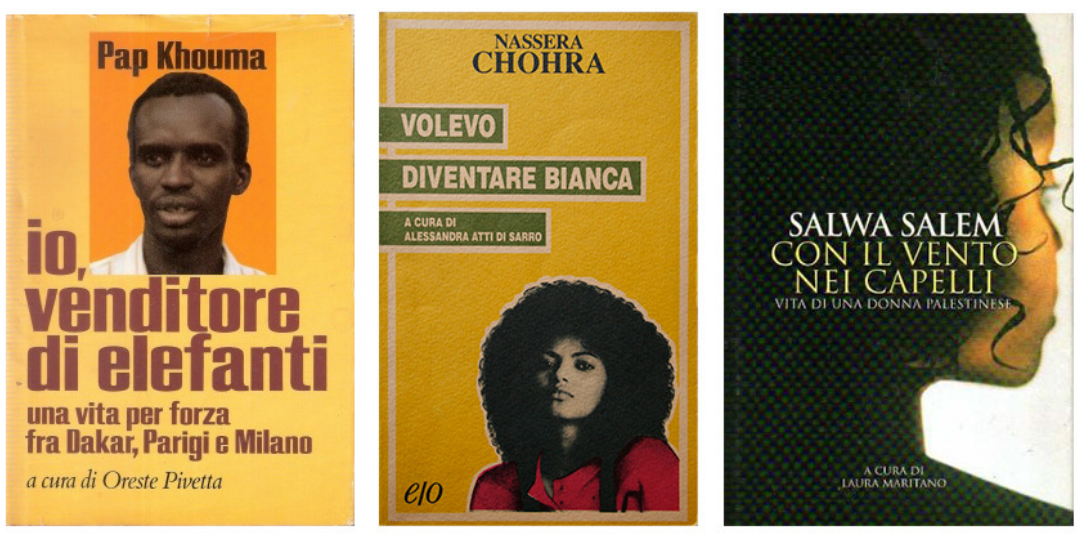

Fig. I. Couvertures des romans Io venditore di elefanti (Pap Khouma et Oreste Pivetta, Garzanti, 1990), Volevo diventare bianca, (Nassera Chohra et Alessandra Atti di Sarro, E/O, 1993), et Con il vento nei capelli (Salwa Salem et Laura Maritano, Giunti, 1990).

En somme, le paratexte - toujours riche, en quatrième de couverture, d'indications biographiques qui garantissent la coïncidence de l'auteur immigré avec les événements racontés - guide d'emblée le lecteur vers la reconnaissance d'une

${ }^{21}$ C'est le cas par exemple de Carla De Girolamo et Daniele Miccione dans Mohamed Bouchane, Chiamatemi Ali. Carla De Girolamo et Daniele Miccione (dir.), Milano, Leonardo, 199I; de Laura Maritano dans Salwa Salem, Con il vento nei capelli, Laura Maritano (dir.), Milano, Giunti, 1990; d'Oreste Pivetta dans Pap Khouma, Io venditore di elefanti, Oreste Pivetta (dir.), Milano, Garzanti, I990; et d'Alessandra Atti di Sarro dans Nassera Chohra, Volevo diventare bianca, Alessandra Atti di Sarro (dir.), Roma, E/O, 1993. 
division du travail qui voit d'un côté l'étranger comme porteur d'expérience personnelle et directe, et, de l'autre, l'Italien comme garant d'une langue et d'une tradition littéraire. S’amorce ainsi un court-circuit selon lequel celui qui est présenté comme auteur (suivant de spécifiques stratégies de marché) ne détient cependant pas l'autonomie et l'autorité de la parole, et se retrouve de ce fait du côté de « l'informateur indigène » (native informant) dont parle Spivak ${ }^{22}$, puisque ses mots sont sans cesse réécrits, transcrits, traduits.

Voilà sans doute une typologie de traduction que l'on peut difficilement associer aux traditionnelles procédures traductives intertextuelles, car le texte d'origine est presque toujours difficile à repérer. C'est en fait plutôt une traduction in absentia ${ }^{23}$, dont l'original n'est pas perdu, comme c'était le cas pour de nombreux textes anciens, mais existe à l'oral ou bien se configure comme carnet de notes éparses, ou encore comme série d'enregistrements vocaux.

De plus, il s'agit d'une modalité traductive intralinguistique ou endolinguistique, c'est-à-dire d'une traduction dans une même langue: de l'italien (déjà traduit) de l'auteur migrant à l'italien de l'auteur autochtone, qui ne sont pas les mêmes langues, puisqu'elles ne caractérisent pas la même «communauté », ne renvoient pas aux mêmes compétences, ni aux mêmes habitudes linguistiques. La première, la migrante, a besoin, semble-t-il, de la seconde pour être comprise et acceptée par la communauté littéraire; la deuxième, l'autochtone, joue dès lors le rôle de « garant » linguistico-littéraire, offrant à la fois une certification de conformité et d'authenticité linguistique, et une licence de publication. Se dessine devant nos yeux un paysage où les frontières d'une langue ne sont pas seulement le résultat d'une construction culturelle historiquement située et géopolitiquement circonscrite (qui tend à coïncider avec la naissance de l'État-nation), mais aussi des lignes de démarcation, des murs de séparation, qui suivent et renforcent d'autres lignes de scission, celles du pouvoir, et qui en tant que telles peuvent se déplacer, se réécrire et, comme c'est le cas ici, tracer des chemins de division internes sur un même territoire sociolinguistique.

Qui plus est, dans cette problématique dynamique «co-auctorielle », où auctorialité et autorité ne coïncident fatalement pas, le statut d'original et celui de texte traduit se dissolvent jusqu'à se superposer et, en même temps, s'opposer, amorçant un deuxième court-circuit, strictement formel cette fois, celui entre autobiographie et

${ }^{22}$ Voir Gayatri Chakravorty Spivak, A Critique of Postcolonial Reason: Toward a History of the Vanishing, Calcutta, Seagull Books, 1999.

${ }_{23}$ Voir à ce propos: Loredana Polezzi, «Questioni di lingua: tra traduzione e autotraduzione », dans Pezzarossa et Rossini, 2oII, p. 2I. 
hétérobiographie. Si les dispositifs et les modalités de transcription et de traduction peuvent varier d'un texte à l'autre, tous se présentent en effet comme des témoignages autobiographiques et, simultanément, des flux ininterrompus de «narration spontanée », soit des récits dans lesquels la figure de l’ «éditeur-traducteur » et la pratique traductive ont été soigneusement effacées par voie d'appropriation: l'« éditeur-traducteur » italien parle comme s'il était l'auteur étranger, comme s'il racontait sa propre histoire. Mario Fortunato, auteur d'Immigrato avec Salah Methnani, ne se gêne d'ailleurs pas pour le reconnaître :

Je l'avais écrit comme s'il s'agissait de mon histoire. Avec une adhésion sincère, presque médiumnique, je déversais l'histoire de Salah comme si c'était la mienne ${ }^{24}$.

L'auteur immigré n'est plus ainsi ni l'auteur ni le co-auteur, mais le personnage de l'histoire, le protagoniste du roman. Nous assistons alors à une forme spécifique et problématique d'appropriation, qui exclut non seulement la langue de l'immigré, comme n'étant pas «suffisamment italienne » ou «suffisamment littéraire », mais également sa voix, transformant l'immigré en personnage : l'immigré ne raconte pas, il est raconté; il ne prend pas la parole, on lui dérobe encore une fois sa voix.

Ce qui nous ouvre un autre paysage encore, celui où les dispositifs mis en œuvre par la construction de frontières linguistiques « dures » (qu'elles soient internes ou externes) et l'action de certaines pratiques traductives peuvent s'agencer jusqu'à réduire l'autre au silence.

L'autre cas que je voudrais parcourir renvoie au phénomène de la co-présence de plusieurs langues dans un texte, phénomène que, grâce à Rainier Grutman ${ }^{25}$ et Myriam Suchet ${ }^{26}$, nous nous sommes habitués à appeler hétérolinguisme et qui fait de la traduction le substrat, la marque presque, de la plupart des textes postcoloniaux.

Si les langues, à travers la pratique de la traduction interlinguistique, se mettent en scène comme différentes et équivalentes, renvoyant à des communautés locutrices également différentes et équivalentes, le cas particulier de l’hétérolinguisme, c'est-à-dire de

24 « Lo avevo scritto come si trattasse di una storia interamente mia. [...] Con sincera, direi perfino un po' medianica adesione, sciorinavo la storia di Salah come si fosse la mia propria storia. » Mario Fortunato, «Introduzione », dans Mario Fortunato et Salah Methnani (dir.), Immigrato, Milano, Bompiani, 2006, p. VI (notre traduction).

25 Rainier Grutman définit l'hétérolinguisme comme « la présence dans un texte d'idiomes étrangers, sous quelque forme que ce soit, aussi bien que de variétés (sociales, régionales ou chronologiques) de la langue principale ». Rainier Grutman, Des langues qui résonnent. L hétérolinguisme au XIX $X^{e}$ siècle québécois, Montréal, Fides, 1997, p. 37.

${ }^{26}$ Myriam Suchet, L'imaginaire hétérolingue. Ce que nous apprennent les textes à la croisée des langues, Paris, Classiques Garnier, 2014. 
la présence d'un dispositif plurilingue au sein d'un même texte, participe à la déconstruction de la forteresse monolingue des langues, nous accompagnant vers l'idée d'une porosité constitutive des langues, de leur transformation et de leur superposition continuelle.

Il est toutefois naïf de penser que les modalités littéraires de l'hétérolinguisme se conforment à des usages spécifiques dans la société, et il est dangereux de croire qu'elles caractérisent une communauté en particulier (encore une fois) homogène et distincte des autres. Même si des pratiques hétérolingues se répandent de plus en plus dans la société, à des niveaux différents et au sein de différentes communautés, le caractère apparemment mimétique et documentaire de l'hétérolinguisme dans les textes est, avant tout, une construction littéraire, une « mise en scène », comme l'écrit Myriam Suchet, bref une figure du texte, qui peut se configurer différemment d'un texte à l'autre.

Regardons ce qui se passe dans deux romans de Ubah Cristina Ali Farah, l'une des écrivains les plus reconnus de la production postcoloniale italienne: Madre piccola $^{27}$ et Il comandante del fiume $e^{28}$ (voir le figure 2).
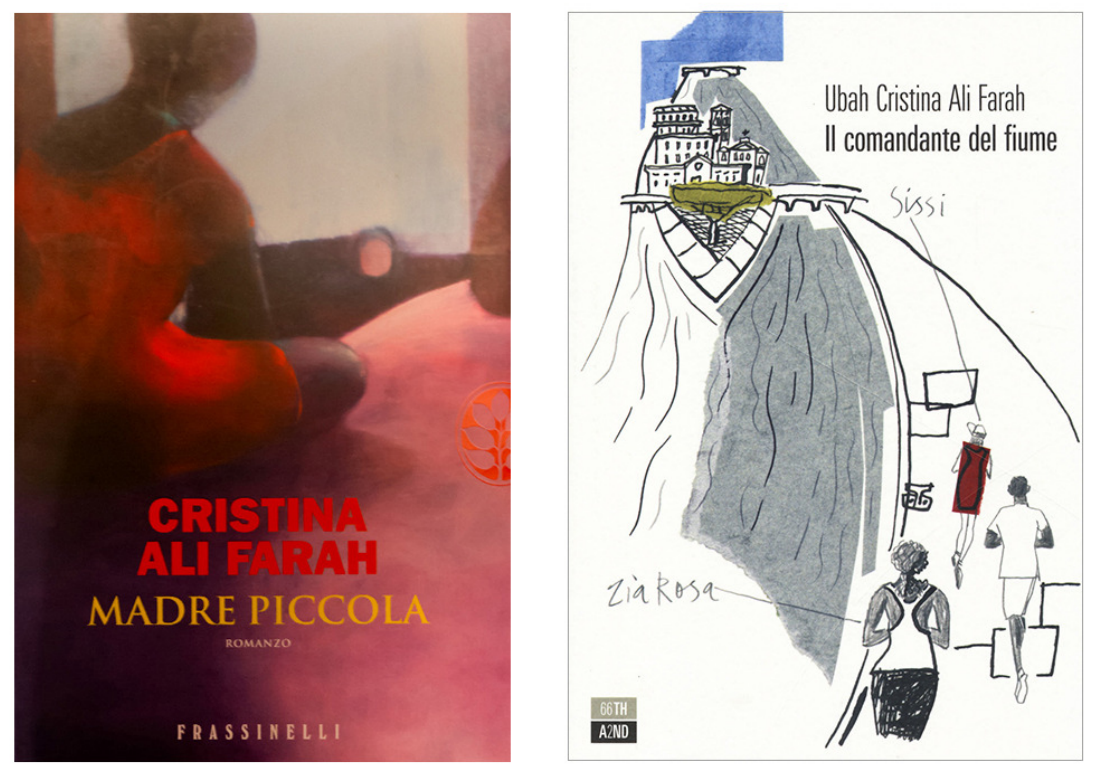

Fig. 2. Couvertures des romans de Ubah Cristina Ali Farah Madre Piccola (Frassinelli, 2007) et Il comandante del fiume (66thand2nd, 2014).

27 Ubah Cristina Ali Farah, Madre piccola, Milano, Frassinelli, 2007.

${ }^{28}$ Ubah Cristina Ali Farah, Il comandante del fiume, Roma, 66thand2nd, 2014. 
Dans ces deux textes, nous assistons à un changement profond quant à la manière d'insérer le lexique somalien et de traiter la rencontre entre les deux langues. Tandis que dans Madre piccola tout mot du somali apparaît en italique, dans Il comandante del fiume le somali se glisse de droit à l'intérieur de l'italien, annulant ainsi toute différence visible entre les deux langues : la différence entre l'italien et le somali est toujours présente à la lecture, certes, créant des zones d'opacité, mais elle est invisible, car aucun indice typographique ne la signale.

En voici trois exemples parmi tant d'autres : « Nous achetions des bajiiye avec du piment frais $\gg^{29}$, «Bajiiye : boulettes de lentilles ${ }^{30} \gg$ ou « [Maman] apporta une boîte de jus de seytuun et un petit plateau de bajiiye ${ }^{3 \mathrm{I}} \gg$.

Ici, le même terme, «bajiiye », est dans le premier roman signalé comme étranger à la langue italienne par l'usage de l'italique et la création d'une entrée dans le glossaire, alors que dans le deuxième roman il est inséré dans le flux de la langue sans qu'aucune indication visible d'altérité ne l'interrompe.

Le degré d' « intégration » des emprunts somaliens est ainsi très différent dans les deux textes : alors que Madre piccola orchestre typographiquement une différence exposée et revendiquée, Il comandante del fiume met en scène un passage silencieux et imperceptible d'une langue à l'autre dans le cadre duquel la différence semble concerner les deux langues à la fois, l'une par rapport à l'autre. En d'autres termes, les différences entre les langues sont annulées dans ce deuxième roman pour ouvrir à la différence de la langue et dans la langue.

Or, la présence d'un glossaire, tout comme celle des notes en bas de page, est le signe le plus manifeste du fait que le mot étranger est introduit (mis en scène) et perçu comme profondément autre, ce qui présente aussi, sur le plan du discours, au moins un inconvénient : celui de mettre en échec le dispositif littéraire cassant le régime de l'énonciation. À l'intérieur du texte s'ouvre une fissure, une faille, qui relève plus du paratexte et qui, écrit de plus Genette ${ }^{22}$, caractérise souvent des textes marqués par une «fonctionnalité très impure » : des textes en équilibre instable entre la fiction et le document dans lesquels les données non fictionnelles tendent à l'emporter. C'est ce qui semble se passer dans les textes migrants italiens, que l'on assigne depuis le début à cette zone floue et problématique dans laquelle non seulement témoignage et narration

\footnotetext{
29 « Compravamo bajiiye con il peperoncino fresco ». Ali Farah, 2007, p. 28.

30 « Bajiiye: polpette di lenticchie », Ibid, p. 269.

${ }^{31}$ « $[$ Mamma $]$ portò una confezione di succo di seytuun e un vassoietto di bajiiye », Ali Farah, 20I4, p. 56.

${ }^{32}$ Gérard Genette, Seuils, Paris, Seuil, 1987, p. 305.
} 
littéraire se mêlent, mais au sein de laquelle témoignage et narration littéraire parfois entrent en conflit, semblent s'exclure.

Or, je ne crois pas que, dans le laps de temps qui sépare les deux romans d'Ali Farah, le niveau d'étrangeté des deux langues ait changé au sein de la société italienne; je crois plutôt que ce qui a changé, c'est la scénographie littéraire, c'est-à-dire la manière de mettre en scène les rapports entre les deux langues et, avec cela, également, le statut (et l'autorité littéraire) des écritures migrantes et de leurs auteurs.

Je veux dire par là que le degré majeur d'«intégration » de la langue somalienne - à savoir la visibilité mineure dont jouissent l'insertion, la variation ou la transgression somalienne - est le symptôme d'une construction littéraire qui, par le choix de l'hétérolinguisme, force de l'intérieur le bastion monolingue. Et cette posture - linguistique, littéraire et politique à la fois - a des effets sur les rapports entre les langues non tant (non seulement) pour les renverser, mais pour les repenser et les reconfigurer. Autrement dit, on assiste à la configuration d'un espace, littéraire, dans lequel à la différence entre les langues - à leur essentialisation, à leur lutte pour le « pouvoir/maîtrise » - on substitue un espace au-delà de l'essentialisation, de la lutte et de la différence. Il s'agit également d'une pratique, d'une posture, qui a un effet immédiat sur le statut de l'acte littéraire: cette dernière sauvegardant l'unité et la continuité de l'énonciation, à savoir la stabilité de la structure fictionnelle du discours, le poids du texte se déplace beaucoup plus du côté de la littérature - de la création, de l'invention littéraire -- que de celui du témoignage et de la réalité.

Le pouvoir de talking back du texte postcolonial hétérolingue consiste alors non seulement en la possibilité de réappropriation, de la part du subalterne, de la langue du «colon », mais aussi (et surtout) en sa capacité de contester et transgresser les frontières linguistiques et culturelles nationales jusqu'à ouvrir à (et en même temps dépasser) la différence, ce qui signifie avant tout déconstruire et transgresser l'idée que la différence distingue et divise.

Si on relit dans cette perspective la déclaration de Dany Laferrière par laquelle nous avons commencé notre réflexion, nous pouvons nous en saisir, nous l'approprier, pour mélanger davantage les cartes et questionner l'idée (et l'existence) d'une langue maternelle, tout comme celle d'une langue, conçue comme un système stable, clos et immuable, distinct d'un autre système (d'une autre langue) également stable, clos et immuable. Cette idée reproduit la même essentialité et la même organisation binaire des rapports entre les peuples et les cultures sur laquelle s'est fondée tant la construction (l'invention) des États-nations que l'entreprise coloniale, et sur laquelle se base, aujourd'hui, toute la rhétorique du choc de civilisations. 


\title{
Traduction, re-traduction, hétérolinguisme dans la littérature postcoloniale italienne
}

\author{
lucia Quaquarelli, Université Paris-Ouest Nanterre la \\ DÉFENSE
}

\section{RÉSUMÉ}

La littérature postcoloniale italienne interroge le statut du texte tant sur le plan de son appartenance à un auteur et à une tradition ou canon littéraires nationaux, que sur le plan de l'homogénéité de la langue, des rapports de pouvoir entre les langues (et les littératures) et les stratégies de traduction. Le cas de Immigrato (1990) de Mario Fortunato et Salah Methnani, ainsi que les romans de Ubah Cristina Ali Farah, Madre piccola (2007) et Il comandante del fiume (2014), nous permettront de discuter ces hypothèses.

\section{ABSTRACT}

Italian postcolonial literature questions the status of the text from the point of view of its belonging to an author's oeuvre and to a national literary tradition/canon, as well as from the perspective of homogeneity of language, power relations between languages (and literatures), and strategies of translation. The case of Immigrato (1990) by Mario Fortunato and Salah Methnani, as well as Ubah Cristina Ali Farah's novels Madre piccola (2007) and Il comandante del fiume (2014), will help us discuss these hypotheses.

\section{NOTE BIOGRAPHIQUE}

LUCIA QUAQUARELL enseigne la théorie et la méthodologie de la traduction à l'Université Paris Nanterre. Elle traduit aussi des romans et étudie les formes du récit contemporain. Parmi ses publications récentes sont Narrazione e migrazione (Morellini, 20I5) et Bande à part. Graphic Novel, fumetto e letteratura (Morellini, 2016, codirigé avec Sara Colaone). 